\title{
Special Issue: Recent Advances in Acoustic Black Hole Research
}

\section{Editorial}

F. Gautier $^{(1)}$, V.V. Krylov ${ }^{(2)}$

(1) Laboratoire d'Acoustique de L'Universite du Mans (UMR CNRS 6613), Av. O. Messaien 72085, Le Mans cedex 9, France

(2) Department of Aeronautical and Automotive Engineering, Loughborough University, Loughborough, Leicestershire LE11 3TU, UK

Reduction of undesirable structural vibrations and of the associated structure-borne noise is a classical problem that remains important today. There are many ways of passive damping of structural vibrations. Unfortunately, most of them require addition of substantial amounts of damping materials, which is not always possible because of the mass and volume restrictions associated with structures' operational requirements. In this connection, development of new methods of vibration damping is always desirable, especially if they can avoid the mass or volume penalty. Among such new methods are those based on the Acoustic Black Hole effect $(\mathrm{ABH})$, which is a topic of increasing interest in the vibroacoustic and noise control communities. In the majority of practical situations, this innovative technique for reduction of structural vibrations and of the associated noise is based on the propagation properties of flexural (bending) waves in thin structures of variable thickness. If a local thickness of a structure changes with wave propagation distance quickly enough, then such a structure may result in trapping the vibrations in the area of progressively decreasing thickness, in which a small patch of viscoelastic coating is sufficient to dissipate the localized energy very efficiently.

The scientific literature on this subject has grown rapidly in recent years. In particular, a large number of research papers have been published in leading international journals (Journal of Sound and Vibration, Journal of the Acoustical Society of America, Applied Acoustics, Journal of vibration and acoustics, Mechanical Systems and Signal Processing, Acoustical Physics, Acta Acustica United with Acustica etc.) by teams based in Europe (UK, France, Spain, Germany), the United States, Russia and Asia (China, South Korea). Since 2015, special congress sessions dedicated to this topic are also organised annually at the international conferences 'Inter-noise'.

The community of researchers active in this field was brought together at the ABH2018 workshop organized in May 2018 in Le Mans University, France (https://abh2018.sciencesconf.org), 30 years after the publication of the first article on the topic by M. Mironov. During this event, the publication of a special issue of the Journal of Sound and Vibrations was deemed relevant to give more visibility to this topic of growing interest. A major part of the participants in this workshop gave their commitment to produce original articles in such a special issue. 
This Virtual Special Issue (VSI) aims to present the views of leading international experts in the Acoustic Black Hole research and its applications on the current status of this problem and on its further developments. The ABH literature today, as in March 2020, consists of around a hundred peer reviewed journal papers (in addition to numerous conference papers), among them 40 papers have been published in the Journal of Sound and Vibration (JSV). This Virtual Special Issue of JSV includes 11 papers listed below as references [1-11]. For convenience of the readers, references to other ABH papers published in JSV, that are not part of this VSI, are also provided in the same Reference List as references [12-40].

The first paper in the List is a review paper [1], which provides a discussion of the current status of the theory and applications of the $\mathrm{ABH}$ strategy. It should be noted that $\mathrm{ABH}$ techniques have been developed and traditionally employed to reduce structural vibrations and the resulting structure-borne noise, especially in light-weight structures. However, more recently, applications of $\mathrm{ABH}$ to other areas, including elastic metastructures, energy harvesting, vibro-impact systems and cochlear systems, have also been investigated. The great variety of modelling approaches and the numerous design versions around the basic configuration show the richness of this research topic. A second review paper [2] is focused on the modelling of localised flexural waves in elastic wedges of power-law profile and on their relationship with the $\mathrm{ABH}$ effect. Applications of fractional order operators to the description of acoustic ducts with $\mathrm{ABH}$ terminations are considered in the paper [3]. Semianalytical models based on WKB methods are discussed in paper [4] with regard to their ability to predict characteristics of traditional types of $\mathrm{ABH}$ employing beams or plates of variable thickness.

It is known that acoustic black holes are most effective in the high-frequency range, where the wavelength is typically less than its own size. Several strategies have been proposed to overcome this limitation. The first one is based on the numerical optimisation of the coating layer [5]. Another one applies the damping control in the central zone of ABH. In particular, in paper [6], it is proposed to control the temperature of the central zone in order to tune the damping. This directly impacts on the $\mathrm{ABH}$ performances in the whole frequency range. Among other possibilities proposed to improve the $\mathrm{ABH}$ performance at low frequencies, a vibro-impact strategy has been tested [7]. It induces contact non-linearities in the central zone, which converts the components of the input signal from the low to the high frequencies to improve the ABH damping performance. Paper [8] describes the design of phononic plates based on a periodic lattice of acoustic black holes and capable of supporting topological edge states. The design is based on the established concept of the acoustic valley Hall effect, which is the elastic analogue of the quantum valley Hall effect for quantum mechanical systems.

A new method for wide-field vibrometry based on high-speed digital holographic interferometry is presented in paper [9]. Using this method, experimental investigation of travelling acoustic waves propagating in alloy plate equipped with a two-dimensional $\mathrm{ABH}$ is considered. Paper [10] presents the results of the experiments on two different waveguide models of $\mathrm{ABH}$ for air-borne sound and compares the results with the exact analytical solutions of the wave propagation equations that can be obtained for both models. Paper [11] proposes a practical improvement to the traditional $\mathrm{ABH}$ beam structure by wrapping it up into a spiral. The resulting $\mathrm{ABH}$ structure then adopts a compact shape, which makes it easier to be integrated into different mechanical engineering applications.

Resuming the above, this VSI provides a state of the art in the recent ABH research. Future developments in this area can be expected in combination of $\mathrm{ABH}$ techniques with other physical effects. For example, design of periodic lattices of $\mathrm{ABH}$, using $\mathrm{ABH}$ with piezoelectric patches, their association with nonlinear and vibro-impact phenomenae, thermal control of $\mathrm{ABH}$ to modify damping, etc. The $\mathrm{ABH}$ strategy is very rich, and so far has been 
studied mostly in academic contexts. Engineering adaptations and proposals still need to be developed to enable its effective integration into industrial applications.

\section{References}

[1] A. Pelat, F. Gautier, S.C. Conlon, and F Semperlotti. The acoustic black hole: a review of theory and applications. Journal of Sound and Vibration, 2020 (in press).

[2] V.V. Krylov. Overview of localised flexural waves in wedges of power-law profile and comments on their relationship with the acoustic black hole effect. Journal of Sound and Vibration, 468: 115100, 2020.

[3] J.P. Hollkamp and F. Semperlotti. Application of fractional order operators to the simulation of ducts with acoustic black hole terminations. Journal of Sound and Vibration, 465: 115035, 2020.

[4] A. Karlos, S.J. Elliott, and J. Cheer. Higher-order WKB analysis of reflection from tapered elastic wedges. Journal of Sound and Vibration, 449: 368-388, 2019; Corrigendum, Journal of Sound and Vibration, 467: 115059, 2020.

[5] L. Ma and L. Cheng. Topological optimization of damping layout for minimized sound radiation of an acoustic black hole plate. Journal of Sound and Vibration, 458: 349$364,2019$.

[6] M. Ouisse, D. Renault, P. Butaud, and E. Sadoulet-Reboul. Damping control for improvement of acoustic black hole effect. Journal of Sound and Vibration, 454: 63-72, 2019.

[7] H. Li, C. Touze, A. Pelat, F. Gautier, and X. Kong. A vibro-impact acoustic black hole for passive damping of flexural beam vibrations. Journal of Sound and Vibration, 450: 28-46, 2019.

[8] S.S. Ganti, T.-W. Liu, and F. Semperlotti. Topological edge states in phononic plates with embedded acoustic black holes. Journal of Sound and Vibration, 466: 115060, 2020 .

[9] L. Lagny, M. Secail-Geraud, J. Le Meur, S. Montresor, K. Heggarty, C. Pezerat, and P. Picart. Visualization of travelling waves propagating in a plate equipped with $2 \mathrm{D} \mathrm{ABH}$ using wide-field holographic vibrometry. Journal of Sound and Vibration, 461:114925, 2019.

[10] M. Mironov and V. Pislyakov. One-dimensional sonic black holes: Exact analytical solution and experiments. Journal of Sound and Vibration, 473:115223, 2020.

[11] S. Park, M. Kim, and W Jeon. Experimental validation of vibration damping using an archimedean spiral acoustic black hole. Journal of Sound and Vibration, 459:114838, 2019.

[12] V.V. Krylov and F.J.B.S. Tilman. Acoustic 'black holes' for flexural waves as effective vibration dampers. Journal of Sound and Vibration, 274(3-5): 605-619, 2004.

[13] V.V. Krylov and R.E.T.B. Winward. Experimental investigation of the acoustic black hole effect for flexural waves in tapered plates. Journal of Sound and Vibration, 300(12): 43-49, 2007. 
[14] D.J. O'Boy, V.V. Krylov, and V. Kralovic. Damping of flexural vibrations in rectangular plates using the acoustic black hole effect. Journal of Sound and Vibration, 329(22): 4672-4688, 2010.

[15] D.J. O'Boy and V.V. Krylov. Damping of flexural vibrations in circular plates with tapered central holes. Journal of Sound and Vibration, 330(10): 2220-2236, 2011.

[16] V.B. Georgiev, J. Cuenca, F. Gautier, L. Simon, and V.V. Krylov. Damping of structural vibrations in beams and elliptical plates using the acoustic black hole effect. Journal of Sound and Vibration, 330(11): 2497-2508, 2011.

[17] V. Denis, A. Pelat, F. Gautier, and B. Elie. Modal overlap factor of a beam with an acoustic black hole termination. Journal of Sound and Vibration, 333(12):2475-2488, 2014.

[18] S. Foucaud, G. Michon, Y. Gourinat, A. Pelat, and F. Gautier. Artificial cochlea and acoustic black hole travelling waves observation: Model and experimental results. Journal of Sound and Vibration, 333(15): 3428-3439, 2014.

[19] V. Denis, F. Gautier, A. Pelat, and J. Poittevin. Measurement and modelling of the reflection coefficient of an acoustic black hole termination. Journal of Sound and Vibration, 349: 67-79, 2015.

[20] V. Denis, A. Pelat, and F. Gautier. Scattering effects induced by imperfections on an acoustic black hole placed at a structural waveguide termination. Journal of Sound and Vibration, 362: 56-71, 2016.

[21] L. Tang, L. Cheng, H. Ji, and J. Qiu. Characterization of acoustic black hole effect using a one-dimensional fully-coupled and wavelet-decomposed semi-analytical model. Journal of Sound and Vibration, 374: 172-184, 2016.

[22] O. Aklouche, A. Pelat, S. Maugeais, and F. Gautier. Scattering of flexural waves by a pit of quadratic profile inserted in an infinite thin plate. Journal of Sound and Vibration, 375: 38-52, 2016.

[23] L. Zhao. Embedded acoustic black holes for broadband semi-passive vibration attenuation using shunted pzt in thin-walled structures. Journal of Sound and Vibration, 388: 42-52, 2017.

[24] M.K. Kalkowski, J.M. Muggleton, and E. Rustighi. An experimental approach for the determination of axial and flexural wavenumbers in circular exponentially tapered bars. Journal of Sound and Vibration, 390:67-85, 2017.

[25] L. Tang and L. Cheng. Enhanced acoustic black hole effect in beams with a modified thickness profile and extended platform. Journal of Sound and Vibration. 391: 116-126, 2017.

[26] O. Guasch, M. Arnela, and P. Sanchez-Martin. Transfer matrices to characterize linear and quadratic acoustic black holes in duct terminations. Journal of Sound and Vibration, 395: 65-79, 2017.

[27] W. Huang, H. Ji, J. Qiu, and L. Cheng. Analysis of ray trajectories of exural waves propagating over generalized acoustic black hole indentations. Journal of Sound and Vibration, 417: 216-226, 2018.

[28] L. Ma, S. Zhang, and L. Cheng. A 2D Daubechies wavelet model on the vibration of rectangular plates containing strip indentations with a parabolic thickness profile. Journal of Sound and Vibration, 429: 130-146, 2018. 
[29] T. Zhou and L. Cheng. A resonant beam damper tailored with acoustic black hole features for broadband vibration reduction. Journal of Sound and Vibration, 430: 174184, 2018.

[30] X. Li and Q. Ding. Sound radiation of a beam with a wedge-shaped edge embedding acoustic black hole feature. Journal of Sound and Vibration, 439: 287-299, 2019.

[31] L. Zhao. Low-frequency vibration reduction using a sandwich plate with periodically embedded acoustic black holes. Journal of Sound and Vibration, 441: 165-171, 2019.

[32] W. Huang, H. Zhang, D.J. Inman, J. Qiu, C. Cesnik, and H. Ji. Low reflection effect by 3D printed functionally graded acoustic black holes. Journal of Sound and Vibration, 450: 96-108, 2019.

[33] P. Zeng, L. Zheng, J. Deng, A. Elsabbagh, S. Xiang, T. Yan, and Y.Wu. Flexural wave concentration in tapered cylindrical beams and wedge-like rectangular beams with power-law thickness. Journal of Sound and Vibration, 452: 82-96, 2019.

[34] J.Y Lee and W. Jeon. Exact solution of Euler-Bernoulli equation for acoustic black holes via generalized hypergeometric differential equation. Journal of Sound and Vibration, 452:191-204, 2019.

[35] H. Ji, X. Wang, J. Qiu, L. Cheng, Y. Wu, and C. Zhang. Noise reduction inside a cavity coupled to a flexible plate with embedded 2-D acoustic black holes. Journal of Sound and Vibration, 455: 324-338, 2019.

[36] J. Deng and O. Guash. Ring-shaped acoustic black holes for broadband vibration isolation in plates. Journal of Sound and Vibration, 458: 109-122, 2019.

[37] C.A. McCormick and M.R. Shepherd. Design optimization and performance comparison of three styles of one-dimensional acoustic black hole vibration absorbers. Journal of Sound and Vibration, 470: 115164, 2020.

[38] J. Leng, V. Romero-Garcia, A. Pelat, R. Pico, J-P. Groby, and F. Gautier. Interpretation of the acoustic black hole effect based on the concept of critical coupling. Journal of Sound and Vibration, 471:115199, 2020.

[39] T. Zhou, J.D. Chazot, E. Perry-Debain and L. Cheng. Partition of unity finite element method for the modelling of acoustic black hole wedges. Journal of Sound and Vibration, 475: 115266, 2020.

[40] J. Deng, O. Guasch, L.Zheng, A semi-analytical method for characterizing vibrations in circular beams with embedded acoustic black holes, Journal of Sound and Vibration, 2020, (in press). 